\title{
Risk factor assessment for clinical malaria among forest-goers in a pre-elimination setting in Phu Yen Province, Vietnam
}

\author{
Sara E. Canavati ${ }^{1}$, Gerard C. Kelly ${ }^{1}$, Cesia E. Quintero' ${ }^{1}$, Thuan Huu Vo ${ }^{1}$, Long Khanh Tran ${ }^{1}$, Colin Ohrt ${ }^{1}$, \\ Thang Duc Ngo ${ }^{2}$, Duong Thanh $\operatorname{Tran}^{2}$ and Nicholas J. Martin ${ }^{3^{*}}$ (D)
}

\begin{abstract}
Background: The transition from malaria control to elimination requires understanding and targeting interventions among high-risk populations. In Vietnam, forest-goers are often difficult to test, treat and follow-up for malaria because they are highly mobile. If undiagnosed, forest-goers can maintain parasite reservoirs and contribute to ongoing malaria transmission.

Methods: A case-control study was conducted to identify malaria risk factors associated with forest-goers in three communes in Phu Yen Province, Vietnam. Cases $(n=81)$ were residents from the study area diagnosed with malaria and known to frequent forest areas. Controls $(n=94)$ were randomly selected forest-going residents from within the study area with no identified malaria infection. Participants were interviewed face-to-face using a standard questionnaire to identify malaria risk factors. Logistic regression was used to calculate odds ratios (ORs) and $95 \% \mathrm{Cl}$ for risk factors after adjusting for socio-demographic characteristics.
\end{abstract}

Results: Among the cases, malaria infection varied by species: $66.7 \%$ were positive for Plasmodium falciparum, $29.6 \%$ for Plasmodium vivax, and $3.7 \%$ were diagnosed as mixed infection. Cases were less likely than controls to use treated nets $(\mathrm{aOR}=0.31 ; 95 \% \mathrm{Cl} 0.12-0.80)$, work after dark $(\mathrm{aOR}=2.93 ; 95 \% \mathrm{Cl} 1.35,6.34)$, bath in a stream after dark $(\mathrm{aOR}=2.44 ; 95 \% \mathrm{Cl} 1.02-5.88)$, and collect water after dark $(\mathrm{aOR}=1.99 ; 95 \% \mathrm{Cl} 1.02-3.90)$.

Conclusions: As Vietnam moves toward malaria elimination, these findings can inform behaviour change communication and malaria prevention strategies, incorporating the risk of after-dark and water-related activities, in this priority and difficult-to-access population group.

Keywords: Forest-goers, Risk behaviors, Risk factor analysis, Malaria, Case-control study, Mobile and migrant populations, Malaria elimination, Vietnam

\section{Background}

The malaria burden in Vietnam has been reduced under the Millennium Development Goals and the national malaria programme is now transitioning away from control operations with an aim to eliminate the disease by

\footnotetext{
*Correspondence: Martin.Nicholas.mil@afrims.org

${ }^{3}$ Naval Medical Research Unit TWO, PSA Sembawang Deptford Rd., Building 7-4, Singapore 759657, Singapore

Full list of author information is available at the end of the article
}

$2030[1,2]$. As malaria transmission declines, countries pursuing an elimination agenda must address increased heterogeneity in the distribution of remaining pockets of malaria infection $[3,4]$. Heterogeneity exists both in a geographic context, relating to the spatial distribution of transmission, and in a demographic context, where specific population groups may be at a higher risk of malaria infection or serve as a significant asymptomatic parasite reservoir. To disrupt and halt the potential for ongoing

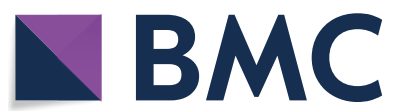

(c) The Author(s) 2019. This article is licensed under a Creative Commons Attribution 4.0 International License, which permits use, sharing, adaptation, distribution and reproduction in any medium or format, as long as you give appropriate credit to the original author(s) and the source, provide a link to the Creative Commons licence, and indicate if changes were made. The images or other third party material in this article are included in the article's Creative Commons licence, unless indicated otherwise in a credit line to the material. If material is not included in the article's Creative Commons licence and your intended use is not permitted by statutory regulation or exceeds the permitted use, you will need to obtain permission directly from the copyright holder. To view a copy of this licence, visit http://creativeco mmons.org/licenses/by/4.0/. The Creative Commons Public Domain Dedication waiver (http://creativecommons.org/publicdomain/ zero/1.0/) applies to the data made available in this article, unless otherwise stated in a credit line to the data. 
transmission, malaria programmes must implement effective strategies that actively seek out, detect and treat all malaria infections, especially in hard to reach groups [5-7].

In the Greater Mekong Subregion (GMS), epidemiological complexities such as the presence of mobile and migrant populations including forest-goers who mostly reside in remote locations, and the spread of anti-malarial drug resistance pose key challenges for malaria programmes $[8,9]$. To address these concerns, Vietnam's elimination programme includes improving access to early diagnosis and prompt effective treatment, ensuring uniform intervention coverage for at-risk populations, and strengthening the malaria epidemiological surveillance system and capacity to implement robust malaria epidemic response [1]. However, even in Vietnam, forestgoers maybe not reached or covered by health services, due in part to their remote location and mobile lifestyles. These characteristics are challenges to effectively test and treat these populations for malaria, as well as track and implement routine malaria prevention measures [10]. If undiagnosed, forest-goers can maintain parasite reservoirs and contribute to ongoing transmission. Due to these factors surveillance systems are not optimized to target these hard to reach groups in Vietnam and the magnitude of this problem is not yet fully elucidated.

To target interventions towards high-risk populations, it is essential for programmes to effectively analyse and identify the associated risk of malaria [11, 12]. In malaria elimination settings, the identification of risk factors becomes difficult using standard methods due to low numbers of infections, calling for novel surveillance approaches [7, 13]. In support of Vietnam's pursuit of malaria elimination, a case-control study was conducted to identify associations between risk behaviours and malaria infection status among forest-goers in Phu Yen Province, Vietnam.

\section{Methods}

\section{Setting and study area}

The study was conducted in Phu Mo, Xuan Lanh, and Xuan Quang 1 communes in Dong Xuan district, northwest Phu Yen Province, located in South-Central Vietnam (Fig. 1). These study sites were selected by the Vietnam National Institute of Malariology, Parasitology and Entomology due to the high malaria burden, a large number of forest-fringe settlements, and their location in bordering two Tier 1 Artemisinin Resistant provinces as defined by the World Health Organization Global Plan for Artemisinin Resistance Containment [14]. The three study communes are the western communes of Dong Xuan District, located directly adjacent to the mountainous and densely forested central highlands region of
Vietnam (Fig. 2). Elevation varies steeply within the study area ranging from approximately 50 metres above sea level in the valleys to more than 1300 metres above sea level in the western mountainous regions. Malaria transmission in Phu Yen occurs primarily from May through November with bimodal peaks in transmission following the rainy seasons $[15,16]$. In 2016, the study area had an approximate population of 18,000 [17]. Due to the characteristics of the province's resources as well as the structure of the occupation, a significant proportion of the study area working population is known to frequently travel to, and sleep in, the forest for their livelihoods [16].

\section{Study design}

A population-based case-control study, matched at the village level, was conducted in the study site from September to November 2016.

The sample size for this case-control study was calculated using the below formula [18].

$$
\mathrm{n}=\frac{\left\{z_{1-\alpha / 2} \sqrt{2 \mathrm{P}_{2}^{*}\left(1-\mathrm{P}_{2}^{*}\right)}+z_{1-\beta} \sqrt{\mathrm{P}_{1}^{*}\left(1-\mathrm{P}_{1}^{*}\right)+\mathrm{P}_{2}^{*}\left(1-\mathrm{P}_{2}^{*}\right)}\right\}^{2}}{\left(\mathrm{P}_{1}^{*}-\mathrm{P}_{2}^{*}\right)^{2}}
$$

The sample size was calculated by assuming an expected ratio of 3 , the prevalence of 0.63 for control group did not exposure to the study risk factor bases on the previous study [19] and case to control ratio of 1:1. With the power of test of $80 \%$ at the significant level of $5 \%$, the sample size needed was 81 cases and 81 controls.

A case was considered as any resident within the three study site communes who met the following criteria: (1) visit to their local commune health facility due to suspected malaria; (2) a positive test for malaria by rapid diagnostic test (RDT; SD BIOLINE Malaria Ag P.f/P.v), and microscopy [20]; (3) willingness and availability to participate in the study; and (4) self-reported history of sleeping overnight in the forest. Those who were not eligible for the study met one or more of the following exclusion criteria: (1) a negative test for malaria by RDT or microscopy; (2) prior malaria diagnosis within the preceding month; (3) taking malaria prophylaxis or treatment in the preceding 2 weeks; (4) clinically diagnosed cases without any diagnostic test for confirmation; or (5) unwillingness or unavailability to participate in the study.

Controls were enrolled within a 2-week time period following identification of the case. As a means to minimize selection bias, controls that were absent were revisited twice before an alternative eligible household member or neighbor was selected. Inclusion criteria for population controls were defined as (1) a negative malaria test by RDT; (2) willingness and availability to participate in the study; (3) resident within the same study site village of the case; and (4) self-reported history of sleeping overnight 


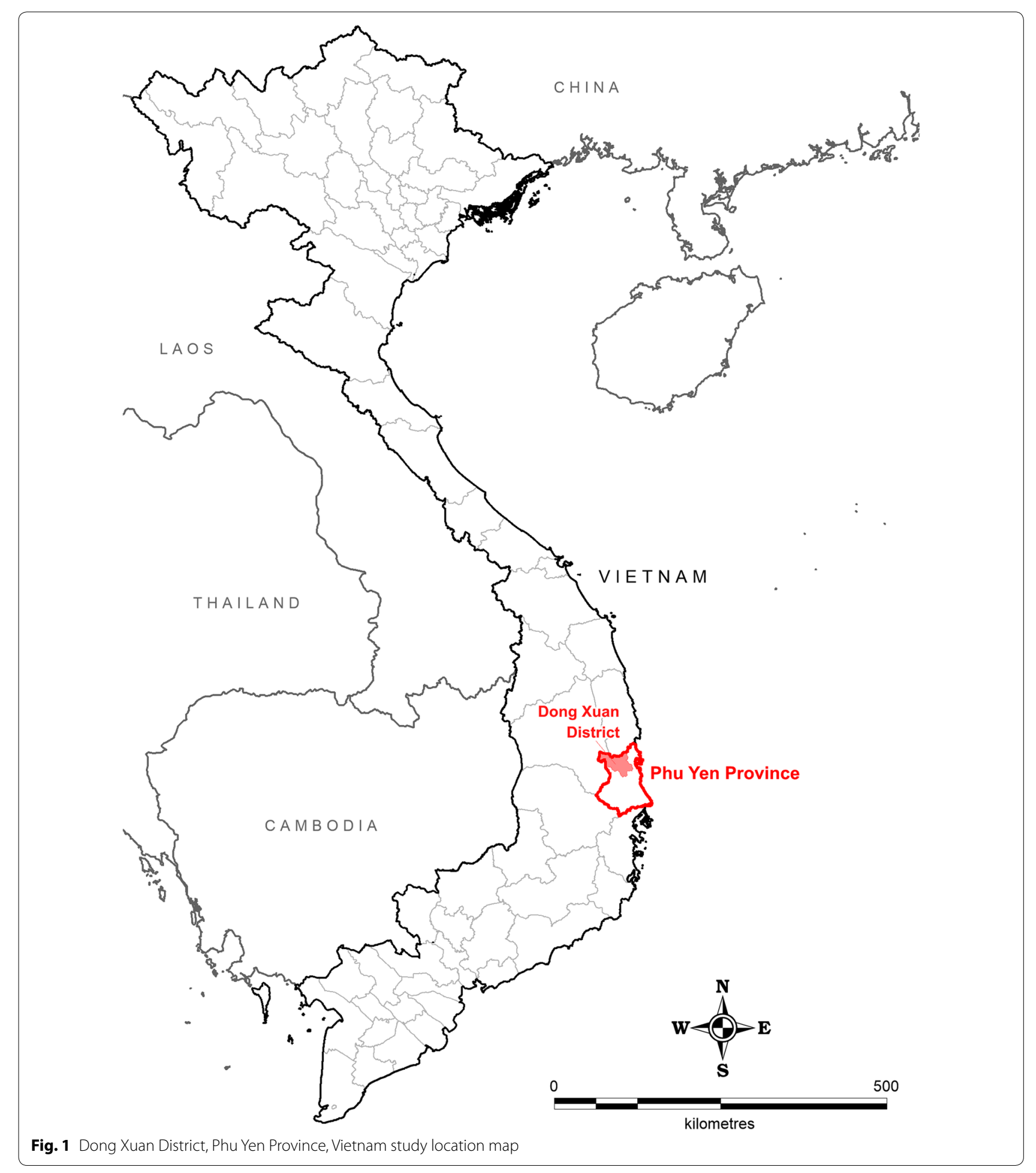

in the forest. Criteria excluding controls included: (1) a positive test for malaria by RDT or microscopy, (2) taking malaria prophylaxis or treatment in the preceding 2 weeks; and (3) unwillingness or unavailability to participate in the study. Controls were randomly selected from the case's home village. All eligible patients that were diagnosed with malaria in the three selected communes during the study period were invited to participate in the study. 


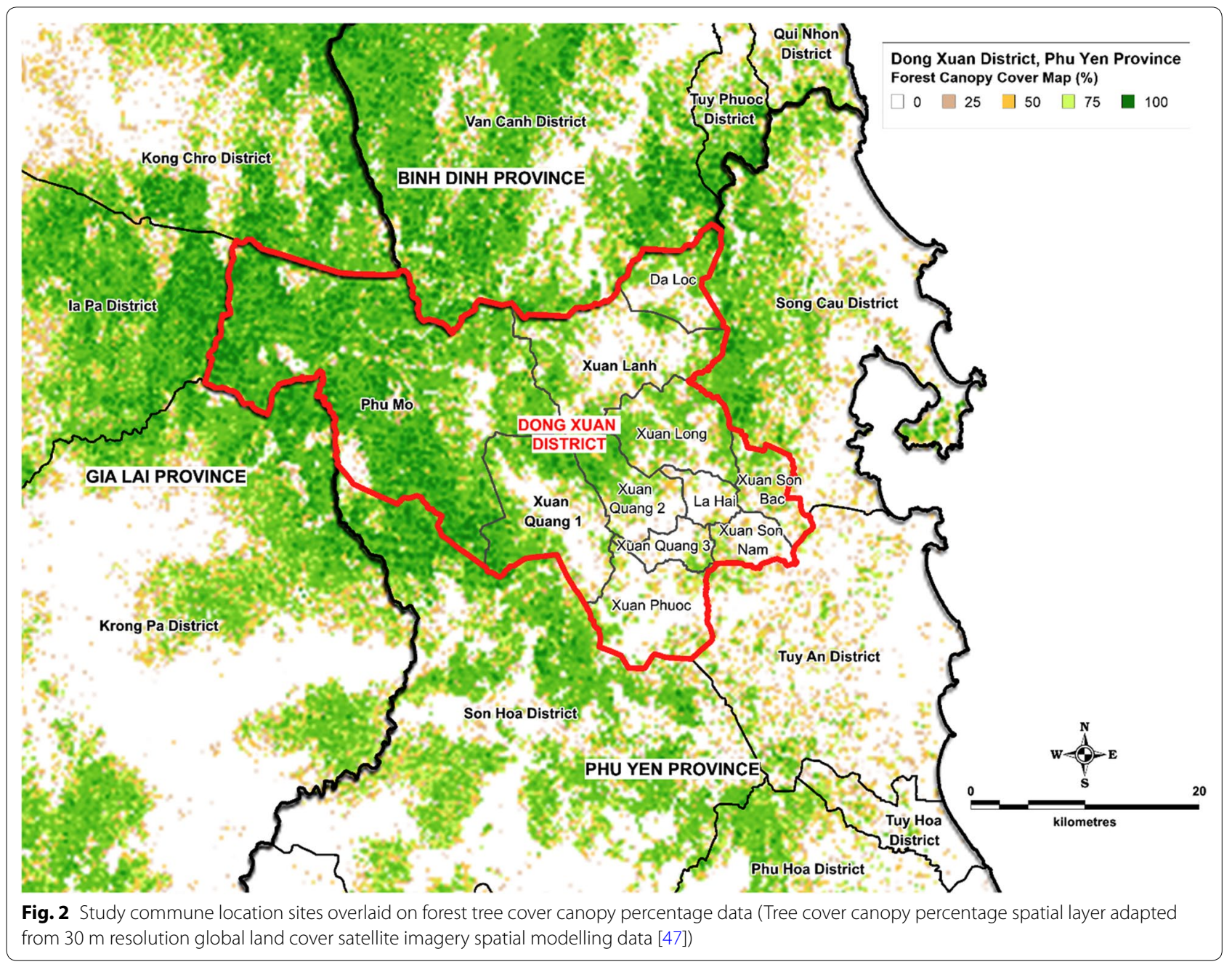

Written informed consent was obtained from all study participants or their legal guardian. All study participants were informed before the start of the interview about the project's goals, the topic and type of questions, the intended use of results for scientific publications, and their right to refuse the interview, interrupt the conversation at any time, and withdraw all given information during or after the interview, without any negative consequences. Anonymity was guaranteed and the confidentiality of interviewees assured by assigning a unique code number to each participant. Local authorities and community leaders were informed of the purpose and expected the duration of the study.

\section{Data collection and procedures}

Cases and controls were interviewed face-to-face using a structured questionnaire (Additional file 1) to capture key socio-demographic data. Data were also collected on behaviour in the forest, including seasonal exposure, sleeping habits, use of hammocks, after-dark activities; and knowledge of and attitudes towards malaria prevention measures. More than one answer could have been accepted per participant, and the answers were categorized accordingly. A standardized questionnaire was developed in English with input from Vietnam National Institute of Malariology, Parasitology and Entomology (NIMPE) staff. It was then translated into Vietnamese, and back-translated into English by a fluent bilingual health expert prior to pre-testing. All members of the study team were trained in standardized interviewing techniques for consistent data collection. Data were captured at the household of participants using smartphones and KLL collect (Kathmandu Living Labs, Nepal) data collection software.

Logic checks, range limits and required answering were programmed into the digital data collection forms as a means to minimize data collection errors. Data were uploaded into an online data storage application (Ona Systems, Nairobi, Kenya) at the point of collection. Study supervisors reviewed and uploaded data daily to ensure 
data quality, completeness and consistency. Data were stored on encrypted via 256 RSA SSL servers with ISO 27001 certification. Answers to the study questionnaires and disease status of participants were kept confidential.

\section{Data analysis}

Data were analysed using $\mathrm{R}$ software (Epi and BMA packages). Chi-square and t-test for normally distributed variables were used to assess statistically significant differences in socio-demographic characteristics between the cases and controls. Social economic status (SES) was defined by quintile technique using principal components and factor analysis of asset score developed from nine asset items, including variables such as electricity, water resources, ownership of motorbike, television, radio, mobile phone, household assets [21]. Crude odds ratios (cORs) and its 95\% confidence intervals (CI) were calculated for risk behaviours of forest-goers between cases and controls by a univariate analysis. The variables that were known to be associated with malaria status from previous studies (including ethnicity, gender, age, education, and SES [22]) were considered in the multivariate logistic regression analysis to calculate the adjusted odd ratios (aORs) for risk behaviours of forestgoers between cases and controls. A 0.05 level of significance was applied for all statistical analysis conducted.

\section{Results}

A total of 90 cases were identified and screened from confirmed malaria cases at the three commune health clinics and 96 neighborhood controls within the study area, which exceeded target enrollment to allow for losses following review of inclusion/exclusion criteria. Out of 186 participants, 11 (nine cases and two controls) were excluded from the study as they did not meet the inclusion criteria. In total 81 cases and 94 controls were enrolled in the study, resulting in an approximate 1:1 case to control ratio. Among the cases, malaria infection varied by species: $66.7 \%$ were positive for Plasmodium falciparum, $29.6 \%$ for Plasmodium vivax, and 3.7\% were diagnosed as mixed infection.

\section{Sociodemographic factors}

Of the 175 enrolled participants, 154 (88.0\%) were male and 147 (84.0\%) had high economic status. Participants were from three ethnic groups: Kinh (34.9\%), Cham (54.9\%) and Bana (10.3\%). Mean age was $36.2 \pm 11.8$ years (Table 1). There were no significant difference with regard to socio-demographic characteristics between cases and controls $(p>005)$, with the exception of age. The mean age of cases $(34.2 \pm 11.7)$ was significantly lower than that of controls $(37.8 \pm 11.6)$. The distribution in gender between cases and controls was not significantly different, males comprised about $88 \%$ of the population for each group. The two highest SES group accounted for a large proportion of the cases (39.5\%) and controls (43.6\%) in this study population.

Among main types of work in the forest, tree planting was not significantly different between cases and controls (53.1\%); however, aloe seeking/hunting (19.8\% compared to $26.6 \%)$ and trapping ( $7.4 \%$ compared to $10.6 \%$ ) were lower in cases than in controls. The proportion of forestgoers who had graduated from secondary school or above was not significantly different between the cases and controls; however, self-reported illiteracy was lower among cases $(22.2 \%)$ compared with controls (29.8\%). Similarly, the proportion of those from the Kinh ethnic group was not significantly different between cases $(34.6 \%)$ and control (35.1\%), but the proportion of Cham and Bana varied between the cases (53.1\% and $12.3 \%)$ and controls $(56.4 \%$ and $8.5 \%$ ), respectively (Table 1 ). Cham ethnic group accounted for more than $80 \%$ of illiterate respondents in this study population $(\mathrm{p}<0.001)$ (Table 2$)$.

\section{Risk factors for malaria}

Risk factors evaluated in this study are presented in Table 3 and were grouped into four general categories: seasonal exposure, sleeping habits, use of nets (including hammock nets), and after-dark activities. Of the risk behaviours investigated in the study, five were significantly associated with malaria. These included: use of treated nets (protective), sleeping in a hut without walls, and participating in after dark activities including collecting water, bathing in streams, and working.

Sleeping with treated nets was associated with significantly lower odds of being a malaria case; the crude OR was 0.31 (95\% CI 0.12-0.77) and did not change after adjusting for age, gender, ethnicity, education level and SES (aOR: 0.31; 95\% CI 0.12-0.80). Using an untreated net or sleeping without any kind of net increased the odds of being a malaria case, however, these association was not statistical significant after adjustment, with the 95\% CI range of 0.44 to 8.09 .

The association between sleeping without any kind of net and the odds of being a malaria case was not significant (cOR 1.16; 95\% CI 0.07-18.88). After adjusting for age, gender, ethnicity, education levels and SES the aOR was 2.21 (95\% CI 0.12-41.08).

There was a significant association between sleeping without any kind of net, sleeping in a hut without walls and the odds of being a malaria case. However, the OR was not computed due to a low number of observations $(\mathrm{n}=4)$.

There was an association between after dark activities and being a malaria case. Collecting water after dark doubled the odds of being a malaria case, with no 
Table 1 Socio-demographic characteristics of cases and controls in Dong Xuan district, Phu Yen province, Vietnam

\begin{tabular}{|c|c|c|c|c|}
\hline Characteristics & Cases $(n=81)(\%)$ & Controls $(n=94)(\%)$ & Total $(n=175)(\%)$ & p-value \\
\hline Age (mean-SD) & $34.2 \pm 11.7$ & $37.8 \pm 11.6$ & $36.2 \pm 11.8$ & 0.04 \\
\hline Gender & & & & 0.90 \\
\hline Male & $71(87.7 \%)$ & $83(88.3 \%)$ & $154(88.0 \%)$ & \\
\hline Female & $10(12.3 \%)$ & $11(11.7 \%)$ & $21(12.0 \%)$ & \\
\hline Education level & & & & 0.54 \\
\hline Illiterate & $18(22.2 \%)$ & $28(29.8 \%)$ & $46(26.3 \%)$ & \\
\hline Primary school & $25(30.9 \%)$ & $21(22.3 \%)$ & $46(26.3 \%)$ & \\
\hline Secondary & $16(19.8 \%)$ & $19(20.2 \%)$ & $35(20.0 \%)$ & \\
\hline High school and above & $22(27.2 \%)$ & $26(27.7 \%)$ & $48(27.4 \%)$ & \\
\hline Ethnicity & & & & 0.70 \\
\hline Kinh & $28(34.6 \%)$ & $33(35.1 \%)$ & $61(34.9 \%)$ & \\
\hline Cham & $43(53.1 \%)$ & $53(56.4 \%)$ & $96(54.9 \%)$ & \\
\hline Bana & $10(12.3 \%)$ & $8(8.5 \%)$ & $18(10.3 \%)$ & \\
\hline Household economic status $^{\mathrm{a}}$ & & & & 0.895 \\
\hline Highest & $17(21 \%)$ & $18(19.1 \%)$ & $35(20 \%)$ & \\
\hline High & $15(18.5 \%)$ & $23(24.5 \%)$ & $38(21.7 \%)$ & \\
\hline Medium & $18(22.2 \%)$ & $17(18.2 \%)$ & $35(20 \%)$ & \\
\hline Low & $17(21 \%)$ & $18(19.1 \%)$ & $35(20 \%)$ & \\
\hline Lowest & $14(17.3 \%)$ & $18(19.1 \%)$ & $32(18.3 \%)$ & \\
\hline Main work in the forest & & & & 0.21 \\
\hline Aloe seeking and hunting & $16(19.8 \%)$ & $25(26.6 \%)$ & $41(23.4 \%)$ & \\
\hline Tree planting & $43(53.1 \%)$ & $50(53.2 \%)$ & $93(53.2 \%)$ & \\
\hline Trapping & $6(7.4 \%)$ & $10(10.6 \%)$ & $16(9.1 \%)$ & \\
\hline Other ${ }^{b}$ & $16(19.8 \%)$ & $9(9.6 \%)$ & $25(14.3 \%)$ & \\
\hline
\end{tabular}

${ }^{a}$ By Quintile

b Making charcoal and logging

Table 2 Education levels of cases and controls by ethnic group

\begin{tabular}{|c|c|c|c|c|c|c|}
\hline Ethnicity & Illiterate $(n=46)(\%)$ & Primary $(n=46)(\%)$ & $\begin{array}{l}\text { Secondary } \\
(n=35)(\%)\end{array}$ & $\begin{array}{l}\text { Highschool } \\
(n=48)(\%)\end{array}$ & Total $(n=175)(\%)$ & $p$-value \\
\hline Bana & $6(13.0 \%)$ & 8 (17.4\%) & $3(8.6 \%)$ & $1(2.1 \%)$ & $18(10.2 \%)$ & \\
\hline Cham & 38 (82.6\%) & $33(71.7 \%)$ & $12(34.3 \%)$ & $13(27.1 \%)$ & $96(54.9 \%)$ & $<0.001$ \\
\hline Kinh & $2(4.4 \%)$ & $5(10.9 \%)$ & $20(57.1 \%)$ & $34(70.8 \%)$ & 61 (34.9\%) & \\
\hline
\end{tabular}

confounding by demographic factors over the association identified $(\mathrm{cOR}=2.00,95 \%$ CI $1.07-3.75$; $\mathrm{aOR}=1.99$, 95\% CI 1.02-3.90). Bathing in a stream after dark increased the odds of being a malaria case $(\mathrm{cOR}=2.59$, 95\% CI 1.12-5.99; aOR $=2.44$, 95\% CI 1.02-5.88). Similarly, there was strong association between work after dark and the odds of being a malaria case, people who worked after dark were significantly more likely to be a malaria case compared to who did not work after dark $(\mathrm{cOR}=2.70,95 \%$ CI $1.30-5.63 ; \mathrm{aOR}=2.93,95 \% \mathrm{CI}$ 1.35-6.34).

\section{Discussion}

While the results from this case-control study are largely consistent with similar studies regarding forest-goers and their risk of malaria in the GMS [23-26], this study reveals additional findings, particularly in relation to after dark behaviour for forest goers residing and working in central Vietnam. The ability to validate findings from other studies in the GMS allows for generalization of regional approaches and can provide contrast to approaches and challenges seen in Africa. Given the epidemiological variances and complexities unique to the 
Table 3 Univariate and multivariate analysis of risk factors for malaria

\begin{tabular}{|c|c|c|c|c|}
\hline Risk factors & Cases $(n=81)$ & Controls $(n=94)$ & cOR $(95 \% \mathrm{Cl})$ & aOR $(95 \% \mathrm{Cl})$ \\
\hline \multicolumn{5}{|l|}{ Seasonal exposure } \\
\hline Number of nights slept in the forest per year (mean-SD) & $130.8 \pm 80.8$ & $118.5 \pm 70.4$ & $1.00(1.00-1.01)$ & $1.00(0.99-1.00)$ \\
\hline $\begin{array}{l}\text { Go to the forest during high risk months (October-Novem- } \\
\text { ber and December) }\end{array}$ & $60(45.5 \%)$ & $72(54.5 \%)$ & $0.87(0.44-1.74)$ & $0.76(0.36-1.60)$ \\
\hline \multicolumn{5}{|l|}{ Sleeping habits (in hammock-bed or floor) } \\
\hline Sleep without any kind of net ${ }^{* \#}$ & $1(50 \%)$ & $1(50 \%)$ & & \\
\hline Use treated nets* & $7(24.1 \%)$ & $22(75.9 \%)$ & $0.31(0.12-0.77)$ & $0.31(0.12-0.80)$ \\
\hline Use untreated nets* & $78(47.3 \%)$ & $87(52.7 \%)$ & $2.09(0.52-8.37)$ & $1.89(0.44-8.09)$ \\
\hline \multicolumn{5}{|l|}{ Use net (treated or untreated) for the whole night } \\
\hline Never or sometimes & $13(54.2 \%)$ & $11(45.8 \%)$ & (ref) & (ref) \\
\hline Usually or always & $68(45 \%)$ & $83(55 \%)$ & $0.69(0.29-1.65)$ & $0.62(0.24-1.63)$ \\
\hline Slept in a hut without walls ${ }^{\#}$ & $15(79 \%)$ & $4(21 \%)$ & & \\
\hline Sleep in hammock with an untreated net* & $33(43.4 \%)$ & $43(56.6 \%)$ & $0.82(0.45-1.49)$ & $0.94(0.48-1.84)$ \\
\hline \multicolumn{5}{|l|}{ After dark activities } \\
\hline Collecting water after dark & $57(52.8 \%)$ & $51(47.2 \%)$ & $2.00(1.07-3.75)$ & $1.99(1.02-3.90)$ \\
\hline Bathing in the stream after dark & $72(50.4 \%)$ & $71(49.6 \%)$ & $2.59(1.12-5.99)$ & $2.44(1.02-5.88)$ \\
\hline Work after dark & $26(65 \%)$ & $14(35 \%)$ & $2.70(1.30-5.63)$ & $2.93(1.35-6.34)$ \\
\hline
\end{tabular}

*Multiple response possible

\#OR not computed due to insufficent number of observations

GMS in comparison to African settings related to the challenges of forest malaria and vector biology, region specific studies of this nature are imperative to inform malaria control and elimination strategies. Additionally, this study allows for a better understanding of demographic characteristics and risk factors specific to forestgoers in Phu Yen province, which should be taken into account when designing interventions at the province level.

Of particular note, and contrary to previous findings [26-29] in Vietnam, neither going to the forest during high-risk months $(\mathrm{OR}=0.87)$, nor sleeping more nights in the forest per year $(\mathrm{OR}=1.00)$, were associated with increased malaria infection in this study population. This suggests that other factors, such as after-dark behaviour, in this setting may have more influence on risk of malaria among forest-goers. Expanded epidemiological research is required within the GMS to better understand risk factors associated with seasonal exposure and duration of stay in the forest. Consistent with other studies in the GMS there were significantly more male than female forest-going respondents [24, 30,31], and these data support current knowledge and practices that males should be the primary target for malaria prevention and control efforts among forest-goers.

Overall, there were no statistically significant differences in demographics between the cases and controls in this study with the exception of age. Larger comparative case-control studies are necessary to better understand why age was a risk factor in this study population. Age has also been found to be a risk factor in certain demographics, such as the Jarai ethnic group along the Cambodia-Vietnam border, with young men, in particular, tending to forego certain protective behaviours, such as using bed nets [24]. Should a perceived lack of importance of personal protection or complacency be identified among high-risk groups, such as young forest-going males, it may be necessary to develop alternative messages such as the potential impact of an individual's behaviour, even when asymptomatic, may have on vulnerable groups such as infants, pregnant women and the elderly within their extended communities. Given the heterogeneity of culture among ethnic groups, stratifying such studies by ethnic group could also provide additional insight for tailoring malaria elimination activities for high risk populations.

Respondents who reported sleeping in a hut without walls were five time more likely to contract malaria than those who slept in huts with walls. However, the relatively small number of respondents who reported sleeping in a hut without walls (less than 11\%) limit the interpretation of this finding. Though the number of total participants from this study was small, several recent publications have also highlighted the significant impact sleeping structures have on malaria incidence [32-34]. In a recent systematic review and meta-analysis, residents of 'modern' houses were observed to have a $47 \%$ lower odds of malaria infection and a 45-65\% lower odds of clinical 
malaria, compared to residents of 'traditional' houses in settings across Asia, Africa and Latin America [35]. The building body of evidence, including data from this study ( $\mathrm{OR}=5.1$ for sleeping in a hut without walls), suggests improved and enclosed sleeping structures should be an area to target future interventions [32-42]. This presents clear challenges in the context of mobile and migrant forest-going populations and novel approaches are clearly required to adequately address such a risk in these settings.

Most participants in the study reported using some kind of mosquito net. However, one of the most striking results from this study is that only $8.6 \%$ of cases and $23.4 \%$ of controls reported using treated nets, despite the protective advantages associated with using treated nets. Further research is needed to determine whether the predominant use of untreated nets is due to access, perceived advantages of untreated nets (such as net type, comfort, portability, suitability, durability, or aesthetics), or cultural barriers. Further attention should also be directed towards the suitability and acceptability of insecticide-treated hammock net options given the proportion of participants using untreated hammock nets in this study and external research findings in this area in relation to their potential protective benefits to forestgoing individuals $[43,44]$.

Although after-dark activities including collecting water, bathing and working were carried out by respondents in both groups, cases were significantly more likely to engage in these practices than controls. Correspondingly their risk of infection was more than double that of controls. Further attention should be given to determine the factors attributable to why cases engaged in afterdark activities such as awareness of risk, cultural influences, environmental, and/or occupational practices and constraints. For example, if a specific forest-based occupation workday typically ends after dusk or is located far from a water source, those individuals might have no choice but to carry out essential activities after dark.

The study identified that all participants in both categories belonged to one of three ethnic groups. As reflected in Table 2, there is a strong correlation between ethnic group and literacy levels, with the Cham accounting for more than $80 \%$ of self-report illiterate respondents. Ethnic group risk analysis data can be used to inform future studies with regard to ethnic groups and their association with specific forest occupations, as well as educational and economic status. Targeted strategies including awareness and behaviour change communication (BCC) interventions should obviously also consider these factors during design and delivery. This is particularly crucial given the reducing malaria burden in the GMS will require increasingly targeted and specialized interventions to support elimination objectives.

Employers in Vietnam could also serve a role to integrate study findings into malaria control and prevention practices. It is difficult to identify and target mobile individuals working for short periods of time, however, working with their static employers may prove less challenging. Whilst it is known there are populations of illegal forest workers in Vietnam, a significant proportion of high-risk forest going groups are also engaged in formal employment within forest settings, such as logging, forest plantations and other agriculture work. Previous studies have shown that employers in the GMS understand the financial benefit in keeping their workforce malaria-free, thus may be motivated to implement malaria prevention practices [45]. Identifying these employers could help address issues related to uptake and use of treated nets and sleeping in an enclosed structure, both factors associated with lower risk of malaria infection in this study.

Matching cases with neighbourhood controls minimized bias related to variations in geographical accessibility to a health facility, few statistically significant differences between the cases and controls were found. This speaks to the challenges of conducting research in elimination settings with low incidence. Although the design did not allow for investigation of ecological risk factors, it did provide statistical power to evaluate micro-epidemiological features related to individual and household factors, as well as an efficient method of data collection.

Due to the nature of the study, data relied on selfreporting; results may, therefore, have been subject to both recall and interviewer bias. Since there was no blinding in this study, it is possible participants altered their answers to be more socially desirable or to produce what they felt was the expected outcome. The fact that cases demonstrated health-seeking behaviour by presenting to the clinic, might suggest a differential bias. It is, therefore, possible that there was a misclassification of the outcome. The study also did not determine whether the two groups were exposed to the same level of malaria BCC and whether they had comparable access to bed nets. These factors could have biased the results. Misclassification of malaria status of controls could have also been an issue, as they were screened with RDTs, which have decreased sensitivity for the detection of low blood parasite concentrations observed in asymptomatic malaria [46]. Those with low blood parasite concentrations could have been misclassified as free from malaria. This study was also unable to capture those who seek services in the private sector; the proportion of forest-goers in the study area who present to the private sector is 
unknown. Furthermore, those who were selected as controls may have contracted malaria and been treated by the private sector. In such cases, they would not appear in the public records as malaria patients and would, therefore, have been considered eligible as a control.

\section{Conclusions}

This case-control study provides valuable information on malaria risk factors of forest going individuals in the Dong Xuan district of Vietnam. The case-control method utilized in this study was a cost-effective and flexible approach to study the heterogeneous malaria risk in an area with diminishing, but persistent malaria burden. Further qualitative studies or larger sample size survey would help determine whether barriers to prevention practices affected cases more than controls and if this is related to factors such as the type of forest work. Findings from this study provide a basis to inform future research on forest-goers in Vietnam to ascertain the extent to which access, age, knowledge or attitude impact the engagement of high-risk behaviours. Given that the use of treated nets was found to be protective and sleeping in a hut without walls was a significant risk factor to contract malaria, further investigation of these risk factors is warranted. With age being the only significant demographic difference, future studies can help determine if younger groups engage in riskier behaviours. Furthermore, these findings can inform future interventions to optimally target, at-risk and heterogeneous populations in malaria elimination settings. Data on risk factors and knowledge can be integrated into national initiatives aimed to combat malaria, both within the public and private sector. This study yields valuable results on risk factors in the Dong Xuan district of Vietnam, both in regards to differences, as well as commonalities, of case and control individuals.

\section{Supplementary information}

Supplementary information accompanies this paper at https://doi. org/10.1186/s12936-019-3068-4.

Additional file 1. Structured questionaire used during face-to-face interviews.

\section{Abbreviations}

BCC: behaviour change communication; Cl: confidence interval; GMS: Greater Mekong Sub-region; NIMPE: Vietnam National Institute of Malariology, Parasitology and Entomology; OR: odd ratio; cOR: crude odd ratio; aOR: adjusted odd ratio; RDT: rapid diagnostic tests; SES: social economic status.

\section{Acknowledgements}

Not applicable.

\section{Authors' contributions}

TDN, CO, DTT, NJM designed the study; TDN and CO collected the data; LKT managed the data; SEC and THV analysed the data; SEC, GCK, CEQ, THV and
NJM wrote the main manuscript text. All authors reviewed the manuscript. Disclaimer for author (NJM) who is U.S. military service member. The views expressed in this article are those of the authors and do not necessarily reflect the official policy or position of the Department of the Navy, the Department of Defense or the U.S. government. All authors read and approved the final manuscript.

\section{Funding}

This work was funded by the U.S. Navy and the U.S. Department of Defense Health Agency Research, Development, Technology and Evaluation programs under work unit number D1423.

\section{Availability of data and materials}

The datasets generated during and/or analysed during the current study are available from the corresponding author on reasonable request.

\section{Ethics approval and consent to participate}

The Institutional Review Board of the NIMPE Protocol number: 2016-VM-P1, The University of California San Francisco Committee on Human Research IRB \#: 14-13540 and Reference \#: 085636) and the NMRC-A Human Research Protection Office (HRPO.NMRCA.2016.0002) approved the study. The methods in this study were carried out in accordance with the relevant guidelines and regulations.

\section{Consent for publication}

All authors and institutes consent to publication. For NJM; I am a military service member or federal/contracted employee of the United States government. This work was prepared as part of my official duties. Title 17 U.S.C. 105 provides that'copyright protection under this title is not available for any work of the United States Government'Title 17 U.S.C. 101 defines a U.S. Government work as work prepared by a military service member or employee of the U.S. Government as part of that person's official duties.

\section{Competing interests}

The authors declare that they have no competing interests.

\section{Author details}

${ }^{1}$ Vysnova Partners, Inc., 4915 St. Elmo Ave., Bethesda 20814, USA. ${ }^{2}$ National Institute of Malariology, Parasitology and Entomology, 35 Trung Van, Hanoi, Vietnam. ${ }^{3}$ Naval Medical Research Unit TWO, PSA Sembawang Deptford Rd. Building 7-4, Singapore 759657, Singapore.

Received: 1 January 2019 Accepted: 9 December 2019

Published online: 20 December 2019

\section{References}

1. NIMPE. National strategy for malaria control and elimination in the period 2011-2020 and orientation to 2030. Vietnam National Institute of Malariology, Parasitology, and Entomology; 2011.

2. UCSF. Country Briefing: Eliminating Malaria in Vietnam. University of California, San Francisco Global Health Group Malaria Elimination Initiative; 2016.

3. Bousema T, Griffin JT, Sauerwein RW, Smith DL, Churcher TS, Takken W. Hitting hotspots: spatial targeting of malaria for control and elimination. PLoS Med. 2012;9:e1001165.

4. Cotter C, Sturrock HJ, Hsiang MS, Liu J, Phillips AA, Hwang J. The changing epidemiology of malaria elimination: new strategies for new challenges. Lancet. 2013;382:900-11.

5. WHO. Global technical strategy for malaria 2016-2030. Geneva: World Health Organization; 2015.

6. Moonen B, Cohen JM, Snow RW, Slutsker L, Drakeley C, Smith DL, et al. Operational strategies to achieve and maintain malaria elimination. Lancet. 2010;376:1592-603.

7. Macauley C. Aggressive active case detection: a malaria control strategy based on the Brazilian model. Soc Sci Med. 2005;60:563-73.

8. Cui L, Yan G, Sattabongkot J, Cao Y, Chen B, Chen X, et al. Malaria in the Greater Mekong Subregion: heterogeneity and complexity. Acta Trop. 2012;121:227-39. 
9. WHO. Eliminatin Malaria in the Greater Mekong Subregion. United to end a deadly disease. Geneva: World Health Organization; 2016.

10. Guyant P, Canavati SE, Chea N, Ly P, Whittaker M, Roca-Feltrer A, et al. Malaria and the mobile and migrant population in Cambodia: a population movement framework to inform strategies for malaria control and elimination. Malar J. 2015;14:252.

11. Herdiana H, Cotter C, Coutrier FN, Zarlinda I, Zelman BW, Tirta YK, et al. Malaria risk factor assessment using active and passive surveillance data from Aceh Besar, Indonesia, a low endemic, malaria elimination setting with Plasmodium knowlesi, Plasmodium vivax, and Plasmodium falciparum. Malar J. 2016:15:468.

12. Wen S, Harvard KE, Gueye CS, Canavati SE, Chancellor A, Ahmed B-N, et al. Targeting populations at higher risk for malaria: a survey of national malaria elimination programmes in the Asia Pacific. Malar J. 2016;15:271.

13. Sturrock H, Hsiang M, Cohen J, Smith D, Greenhouse B, Bousema T, et al. Targeting asymptomatic malaria infections: active surveillance in control and elimination. PLoS Med. 2013;10:e1001467.

14. WHO; Global Malaria Programme. Status report on artemisinin resistance. Geneva: World Health Organization; 2014.

15. Bui $H$, Clements A, Nguyen Q, Nguyen M, Le X, Hay S. Social and environmental determinants of malaria in space and time in Vietnam. Int $J$ Parasitol. 2011:41:109-16.

16. General information of Phu Yen province. http://www.phuyen.gov.vn/ wps/portal/home/trang-chu/chi-tiet/gioi-thieu/gioi-thieu-chung.

17. Canavati S, Thuan V, Ngoduc T, Thanh Tran D, Duc Ngo T, Kelly G, et al. A spatial decision support system approach to implementing malaria surveillance as a core intervention activity in high priority areas of Vietnam. In: 66th annual meeting of the American Society of Tropical Medicine and Hygiene (ASTMH), Baltimore; 2017.

18. Lwanga SK, Lemeshow S, WHO. Sample size determination in health studies: a practical manual. Geneva: World Health Organization, IRIS; 1991. https://apps.who.int/iris/handle/10665/40062.

19. Canavati S, Quintero C, Ngo T, Ohrt C, Vo T, Tran D, et al. Targeted reactive investigative approaches at remote area sleeping sites to interrupt malaria transmission in vietnam. In: Annual meeting of the American Society of Tropical Medicine and Hygiene (ASTMH); 2018:576.

20. WHO. Universal access to malaria diagnostic testing: an operational manual. Geneva: World Health Organization; 2011. p. 158.

21. Moser $C$. The asset vulnerability framework: reassessing urban poverty reduction strategies. World Dev. 1998;26:1-9.

22. Heggenhougen $H$, Hackethal V, Vivek P. The behavioural and social aspects of malaria and its control. Geneva: World Health Organization; 2003

23. Peeters Grietens K, Gryseels C, Dierickx S, Bannister-Tyrrell M, Trienekens S, UkS. Characterizing types of human mobility to inform differential and targeted malaria elimination strategies in Northeast Cambodia. Sci Rep. 2015;5:16837.

24. Gryseels C, Peeters Grietens K, Dierickx S, Xuan XN, Uk S, Bannister-Tyrrell $M$. High mobility and low use of malaria preventive measures among the Jarai male youth along the Cambodia-Vietnam border. Am J Trop Med Hyg. 2015;93:810-8.

25. Grietens KP, Xuan XN, Ribera JM, Duc TN, Bortel W, TruongBa N. Social determinants of long lasting insecticidal hammock-use among the Ra-Glai ethnic minority in Vietnam: implications for forest malaria control. PLOS ONE. 2012;7:e29991.

26. Grietens KP, Xuan XN, Bortel WV, Duc TN, Ribera JM, Nhat TB. Low perception of malaria risk among the Ra-glai ethnic minority in south-central Vietnam: implications for forest malaria control. Malar J. 2010;9:23.

27. Abe T, Honda S, Nakazawa S, Tuong TD, Thieu NQ, Hung LX, et al. Risk factors for malaria infection among ethnic minorities in Binh Phuoc, Vietnam. Southeast Asian J Trop Med Public Health. 2009;40:18-29.

28. Erhart A, Thang ND, Van Ky P, Tinh TT, Van Overmeir C, Speybroeck N, et al. Epidemiology of forest malaria in central Vietnam: a large scale crosssectional survey. Malar J. 2005:4:58.

29. Thanh PV, Van Hong N, Van Van N, Van Malderen C, Obsomer V, RosanasUrgell A, et al. Epidemiology of forest malaria in Central Vietnam: the hidden parasite reservoir. Malar J. 2015;14:86.
30. Kounnavong S, Gopinath D, Hongvanthong B, Khamkong C, Sichanthongthip O. Malaria elimination in Lao PDR: the challenges associated with population mobility. Infect Dis Poverty. 2017;6:81.

31. Sluydts V, Heng S, Coosemans M, Van Roey K, Gryseels C, Canier L, et al. Spatial clustering and risk factors of malaria infections in Ratanakiri Province, Cambodia. Malar J. 2014;13:387.

32. Snyman K, Mwangwa F, Bigira V, Kapisi J, Clark TD, Osterbauer B. Poor housing construction associated with increased malaria incidence in a cohort of young Ugandan children. Am J Trop Med Hyg. 2015;92:1207-13.

33. Liu JX, Bousema T, Zelman B, Gesase S, Hashim R, Maxwell C. Is housing quality associated with malaria incidence among young children and mosquito vector numbers? Evidence from Korogwe, Tanzania. PLoS ONE. 2014;9:e87358.

34. Lwetoijera D, Kiware S, Mageni Z, Dongus S, Harris C, Devine G. A need for better housing to further reduce indoor malaria transmission in areas with high bed net coverage. Parasit Vectors. 2013;6:57.

35. Tusting LS, Willey $B$, Lines J. Building malaria out: improving health in the home. Malar J. 2016;15:320.

36. Okumu F. The paradigm of eave tubes: scaling up house improvement and optimizing insecticide delivery against disease-transmitting mosquitoes. Malar J. 2017;16:207.

37. Tusting LS, Bottomley C, Gibson H, Kleinschmidt I, Tatem AJ, Lindsay SW. Housing improvements and malaria risk in sub-Saharan Africa: a multicountry analysis of survey data. PLoS Med. 2017;14:e1002234.

38. Tusting LS, Ippolito M, Kleinschmidt I, Willey B, Gosling R, Dorsey G. The evidence for improving housing to reduce malaria: a systematic review and meta-analysis. Malar J. 2015;14:209.

39. Consensus statement on housing and malaria. Geneva: Roll Back Malaria Housing and Malaria Work Stream; 2015.

40. Ye Y, Hoshen M, Louis V, Seraphin S, Traore I, Sauerborn R. Housing conditions and Plasmodium falciparum infection: protective effect of iron-sheet roofed houses. Malar J. 2006;5:8.

41. Gamage-Mendis A, Carter R, Mendis C, De Zoysa A, Herath P, Mendis K. Clustering of malaria infections within an endemic population: risk of malaria associated with the type of housing construction. Am J Trop Med Hyg. 1991:45:77-85.

42. Lindsay SW. Can improved housing provide additional protection against clinical malaria over current best practice? International Standard Randomised Controlled Trial Number registration; 2014. https://doi. org/10.1186/isrctn02622179.

43. Morel CM, Thang ND, Erhart A, Xa NX, Peeters Grietens K, Xuan Hung L, Thuan LK, Van Ky P, Hung NM, Coosemans M, et al. Cost-effectiveness of long-lasting insecticide-treated hammocks in preventing malaria in South-Central Vietnam. PLoS ONE. 2013;8:e58205.

44. Sochantha T, Van Bortel W, Savonnaroth S, Marcotty T, Speybroeck N, Coosemans M. Personal protection by long-lasting insecticidal hammocks against the bites of forest malaria vectors. Trop Med Int Health. 2010;15:336-41.

45. Canavati SE, Lawpoolsri S, Quintero CE, Nguon C, Ly P, Pukrittayakamee $\mathrm{S}$, et al. Village malaria worker performance key to the elimination of artemisinin-resistant malaria: a Western Cambodia health system assessment. Malar J. 2016;15:282.

46. malERA Consultative Group on Diagnoses and Diagnostics. A research agenda for malaria eradication: diagnoses and diagnostics. PLoS Med. 2011;8:e1000396.

47. Hansen MC, Potapov PV, Moore R, Hancher M, Turubanova SA, Tyukavina A, et al. High-resolution global maps of 21st-century forest cover change. Science. 2013;342:850-3.

\section{Publisher's Note}

Springer Nature remains neutral with regard to jurisdictional claims in published maps and institutional affiliations. 